примере уборки зерновых и силосных культур) / М-во сельского хоз-ва Российской Федерации, Департамент научно-технологической политики и образования. Челябинск, 2010.

Константинов Михаил Маерович, $\partial-p$ техн. наук, проф. кафедры «Механизация ТП в АПК», Оренбургский государственный аграрный университет. Россия.

Глушков Иван Николаевич, канд. техн. наук, доцент кафедры «Электротехнологии и электрооборудование», Оренбургский государственный аграрный университет. Россия.
Рахимжанова Ильмира Агзамовна, $\partial-p$ c.- $x$. наук, доцент, зав. кафедрой «Электротехнологии и электрооборудование», Оренбургский государственный аграрный универcuтет. Россия.

Герасименко Игорь Владимирович, канд. техн. наук, доцент кафедры «Механизация ТП в АПК», Оренбургский государственный аграрный университет. Россия.

460014, г. Оренбург, ул. Челюскиниев, 18.

Тел.: 8 (3532) 77-39-51.

Ключевые слова: пориионная жатка; шнековый делитель; хлебная масса; мобильное энергетическое средство.

\title{
TO THE QUESTION OF JUSTIFICATION OF OPTIMAL PARAMETERS OF THE SCREW DIVIDER OF THE HEADER PORTION
}

Konstantinov Mikhail Maerovich, Doctor of Technical Sciences, Professor of the chair "Mechanization of Technological Processes in AIC”, Orenburg State Agrarian University. Russia.

Glushkov Ivan Nikolaevich, Candidate of Technical Sciences, Associate Professor of the chair "Electrotechnology and Electrical Equipment", Orenburg State Agrarian University. Russia.

Rakhimzhanova Ilmira Agzamovna, Doctor of Agricultural Sciences, Associate Professor, Head of the chair "Electrotechnology and Electrical Equipment”, Orenburg State Agrarian University. Russia.

Gerasimenko Igor Vladimirovich, Candidate of Technical Sciences, Associate Professor of the chair "Mechanization of Technological Processes in AIC”, Orenburg State Agrarian University. Russia.

Keywords: header portion; screw divider; beveled grain stalks, mobile energy means.
The relevance of the portion harvester for harvesting grain crops split method is substantiated; its main structural features and considers the essence of the technological process are described. The article deals with the essence of the process of preventing contact of beveled grain stalks with wheels of the mobile energy means during the operation of the header, as well as the design and technology of the devices used for this purpose. The research on the establishment of the optimal parameters of the screw divider of the portion header, which is the main component of the device for the removal of bread mass, is presented. The established optimal design and operating parameters of the screw divider, which provides the correct operation of the device for the removal of bread mass from the wheels of the mobile device, are presented.

\section{ОБ ОДНОМ АЛГОРИТМЕ ПРОЕКТИРОВАНИЯ МНОГОСЛОЙНЫХ МЕТАЛЛОКОМПОЗИТНЫХ БАЛЛОНОВ ДАВЛЕНИЯ С ТОНКОСТЕННЫМ ЛЕЙНЕРОМ}

\author{
ЛЕБЕДЕВ Игорь Константинович, ООО «Системы армированных фильтров и трубопроводов» \\ МОРОЗ Николай Григорьевич, ООО «Системы армированных фильтров и трубопроводов» \\ КАЛИННИКОВ Александр Николаевич, Межотраслевой инжиниринговый иентр «Компози- \\ ты России» МГТУ им. Н.Э. Баумана, ФГАОУ ВО «Московский государственный технический \\ университет имени Н.Э. Баумана (национальный исследовательский университет)»
}

Рассмотрена постановка задачи рационального проектирования металлокомпозитного баллона давления, предполагающая определение формы образующей баллона, которая обеспечивает допустимый уровень деформируемости лейнера при заданном ресурсе эксплуатации баллона. Показано, что для окончательно выбранной схемы армирования используют метод ступенчатого нагружения с определением напряжения и деформации, возникающих в материалах конструкции при ее нагружении до уровня испытательного давления.

Введение. При изготовлении баллонов с тонкостенным металлическим лейнером толщина стенки последнего составляет 0,1-0,4 от минимальной толщины композиционного материала на днище. Опыт эксплуатации такого рода металлокомпозитных баллонов давления показывает, что при циклическом воздействии внутреннего давления работоспособность баллона определяется деформированным состоянием металлического лейнера при воздействии циклического нагружения. Согласно деформационным критериям типа Коф-
фина-Менсона [1], использующихся для оценки малоцикловой усталости металлов, число циклов нагружения баллона обратно пропорционально величине пластической деформации, развивающейся в металлическом лейнере при нагружении. В связи с этим рассмотрим постановку задачи рационального проектирования металлокомпозитного баллона давления, предполагающую определение формы образующей баллона, которая обеспечивает допустимый уровень деформируемости лейнера при заданном ресурсе эксплуатации баллона. 
В общем случае будем рассматривать композитную оболочку вращения произвольной формы, состоящую из слоев, выполненных из различных композиционных и изотропных материалов, и нагруженную внутренним давлением.

Методика исследований. В силу тонкостенности лейнера для построения алгоритма проектирования рациональной конструкции воспользуемся соотношениями безмоментной теории оболочек [2-10].

Для оболочек давления, у которых полюсное отверстие с радиусом $r_{0}$ закрыто, осевое усилие

$Q_{1}$ примем равным $p r_{0} / 2$, а погонные нормальные усилия $N_{\alpha}, N_{\beta}$ определяются из соотношений [2]

$$
N_{\alpha}=\frac{p R_{2}}{2}, \quad N_{\beta}=\frac{p R_{2}}{2}\left(2-\frac{R_{2}}{R_{1}}\right)
$$

где $p$ - интенсивность внутреннего давления в рассматриваемой оболочке; $R_{1}, R_{2}$ - главные радиусы кривизны оболочки.

Результаты исследований. Напряженнодеформированное состояние лейнера определим соотношениями деформационной теории пластичности [3] в форме, соответствующей методу переменных параметров упругости:

$$
\sigma_{\alpha}=\bar{E}_{S}\left(\varepsilon_{\alpha}+v_{s} \varepsilon_{\beta}\right), \sigma_{\beta}=\bar{E}_{S}\left(\varepsilon_{\beta}+v_{s} \varepsilon_{\alpha}\right),
$$

где $E_{s}$ - секущий модуль, зависящий от интенсивности напряжений;

$$
\begin{gathered}
\bar{E}_{S}=\frac{E_{S}}{1-v_{s}^{2}}, \quad v_{s}=\frac{1}{2}-\frac{E_{S}}{E}\left(\frac{1}{2}-v\right) ; \\
\sigma_{i}=\sqrt{\sigma_{\alpha}^{2}-\sigma_{\alpha} \sigma_{\beta}+\sigma_{\beta}^{2}} ;
\end{gathered}
$$

$v_{s}$ - пластический коэффициент Пуассона.

Меридиональное и кольцевое усилия, действующие в стенке комбинированного баллона, связаны с деформациями баллона [2]

$$
N_{\alpha}=B_{11} \varepsilon_{\alpha}+B_{12} \varepsilon_{\beta}, N_{\beta}=B_{12} \varepsilon_{\alpha}+B_{22} \varepsilon_{\beta},
$$

где $B_{11}=\bar{E}_{S} h_{m}+B_{11}^{k}, \quad B_{22}=\bar{E}_{S} h_{m}+B_{22}^{k}$, $11 B_{12}=v_{S} \bar{E}_{S} h_{m}+B_{12}^{k}, \quad B_{11}^{k}, B_{22}^{k}, B_{12}^{k}$ - соответству2018 ющие коэффициенты жесткости для композиционного материала.

Усилия $N_{\alpha}$ и $N_{\beta}$ возрастают согласно формулам (1) пропорционально давлению. При этом деформации в композитном слое комбинированной оболочки меньше деформаций в чисто ком- позитной оболочке такой же толщины за счет наличия металлического слоя.

На рисунке представлен пример зависимости секущего модуля и пластического коэффициента Пуассона от интенсивности деформаций, построенной для алюминиевого лейнера [3]. Как следует из представленных диаграмм при деформации баллона, превышающей упругую деформацию лейнера, секущий модуль $E_{s}$ резко уменьшается с ростом деформаций и, например, для рассматриваемого случая, уже при деформации 0,5\% составляет 0,28 от исходного модуля упругости материала лейнера. Таким образом, напряжения в лейнере после появления в его материале пластических деформаций при нагружении баллона возрастают медленнее приращения давления.

При этом напряжения в лейнере также снижаются при увеличении модуля упругости и толщины композитного слоя. Для снижения уровня напряженного состояния лейнера естественно потребовать, чтобы внутреннее давление воспринималось в основном композитным слоем. В то же время наличие металлического лейнера приводит к ограничениям по деформациям оболочки при ее циклическом нагружении.

Как известно, величина упругой деформации металлов, используемых для изготовления лейнера, не превышает 0,21-0,3 \%, что значительно меньше предельной деформации (1,5-3 \%) упругих армирующих волокон композиционного материала. Учитывая данное замечание и исходя из критериев типа Коффина-Менсона [1], при проектировании рациональной геометрии оболочки целесообразно установить некоторое предельное значение интенсивности деформаций возникающих в материале лейнера (как правило, 0,30,5 \%) при действии испытательного давления в баллоне и на их основании установить среднее для данного интервала значение секущего моду-

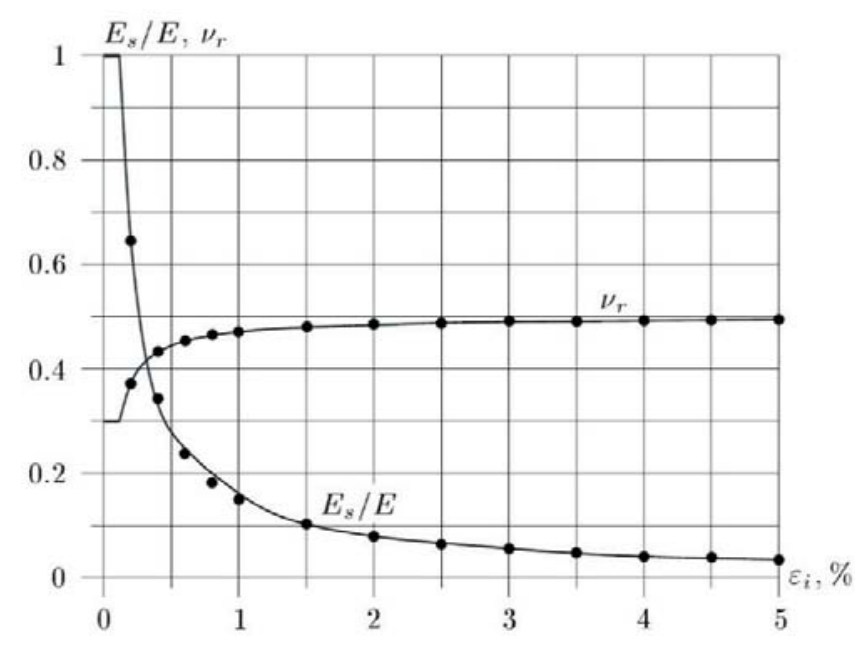

Зависимость секущего модуля и пластического коэффициента Пуассона от интенсивности деформаций 
ля в виде $\bar{E}_{S}=\lambda \bar{E}_{S}^{0},(\lambda=0,8-0,6)$ и использовать его в дальнейшем анализе как постоянную величину. При этом мощность армирования (количество армирующего материала) композитной оболочки следует определять по расчетному давлению с учетом давления, воспринимаемого лейнером, при выбранных, как указано выше, предельных значениях его деформации.

Базируясь на приведенных выше замечаниях, построим алгоритм выбора рациональной формы днищ комбинированного металлокомпозитного баллона давления.

Используя соотношения (1), получим:

$$
\frac{N_{\beta}}{N_{\alpha}}=2-\frac{R_{1}}{R_{2}}
$$

Учитывая, что главные радиусы кривизны оболочки $R_{1}$ и $R_{2}$ могут быть определены с помощью выражений [2]

$$
\frac{1}{R_{1}}=\frac{d \sin \alpha}{d r}, \frac{1}{R_{2}}=\frac{\sin \alpha}{r},
$$

где $\alpha$ - угол между осью вращения и нормалью к поверхности оболочки,

равенство (2) преобразуется к форме

$$
\frac{d \sin \alpha}{\sin \alpha}=\left[2-\frac{N_{\beta}}{N_{\alpha}}\right] \frac{d r}{r} .
$$

Из приведенных соотношений следует, что рациональная форма рассматриваемой безмоментной оболочки определяется отношением действующих внутренних усилий $N_{\beta} / N_{\alpha}$.

Используя соотношения для определения плотности упругой энергии деформации комбинированного материала оболочки при осесимметричном нагружении [2]

$$
U=\frac{N_{\alpha}^{2}}{2} \frac{B_{2}-2 \chi B_{12}+\chi^{2} B_{11}}{B_{22} B_{11}-B_{12}^{2}},
$$

где $\chi=N_{\beta} / N_{\alpha}, B_{i j}$ определяются по соотношениям (2) с учетом $\bar{E}_{S}=\lambda \bar{E}_{S}^{0}$.

Метод множителей Лагранжа [4] для минимизации функционала

$$
\begin{gathered}
F\left(B_{11}, B_{12}, B_{22}\right)=\frac{N_{\alpha}^{2}}{2} \frac{B_{22}-2 \chi B_{12}+\chi^{2} B_{11}}{B_{22} B_{11}-B_{12}^{2}}-\mid \\
-\beta\left(B_{11}+2 B_{12}+B_{22}-C\right)
\end{gathered}
$$

Получим следующее выражение связи отношения действующих внутренних усилий $N_{\beta} / N_{\alpha}$ и механических характеристик $B_{i j}$ материала конструкции.

$$
\frac{N_{\beta}}{N_{\alpha}}=\chi=\frac{B_{22}+B_{12}}{B_{11}+B_{12}} .
$$

Исходя из данного выражения, с учетом выражения для отношения деформаций

$\frac{\varepsilon_{\beta}}{\varepsilon_{\alpha}}=\frac{B_{11} \chi-B_{12}}{B_{22}-B_{12} \chi}$, получим следующий критерий проектирования:

$$
\varepsilon_{\beta}=\varepsilon_{\alpha}=\bar{\varepsilon},
$$

где $B_{i j}$ - коэффициенты жесткости, определяемые с учетом $\bar{E}_{S}=\lambda \bar{E}_{S}^{0} ; \varepsilon_{\alpha}, \varepsilon_{\beta}-$ меридиональная и кольцевая деформации оболочки.

Интенсивность пластических деформаций в материале лейнера

$$
\varepsilon_{\mathrm{i}}^{\mathrm{p}}=\frac{\sqrt{2}}{3} \sqrt{\left[\left(\varepsilon_{\alpha}^{p}-\varepsilon_{\beta}^{p}\right)^{2}+\left(\varepsilon_{\alpha}^{p}-\varepsilon_{h}^{p}\right)^{2}+\left(\varepsilon_{\beta}^{p}-\varepsilon_{h}^{p}\right)^{2}\right]},
$$

где $\varepsilon_{h}^{p}=-\left(\varepsilon_{\alpha}^{p}+\varepsilon_{\beta}^{p}\right)$ - относительное изменение толщины лейнера, в предположении, что объемная пластическая деформация лейнера равна

нулю, при этом будет равна $\varepsilon_{i}^{p}=2 \bar{\varepsilon}$.

То есть критерий проектирования (6) определяет условие равенства деформаций оболочки как в осевом, так и в кольцевом направлении, а также определяет равномерный переход материала лейнера в пластическое состояние с допустимым уровнем пластической деформации, обеспечивающим заданный ресурс эксплуатации баллона.

Алгоритм расчета формы оболочки днищ баллона, базирующийся на соотношении

$\frac{N_{\beta}}{N_{\alpha}}=\frac{B_{12}+B_{22}}{B_{12}+B_{11}}=f(r), \quad$ позволяет проводить оценку оболочки, выполненной как из одного семейства армирующих нитей, так и из многого количества слоев с разными углами армирования в том числе и разнородных материалов.

Для конструкции, изготовленной из одного семейства армирующих нитей без учета металлического лейнера, данное соотношение в развернутой форме принимает следующий вид: 


$$
=\frac{\sin ^{2} \varphi+E_{2} / E_{1} \cos ^{2}}{\cos ^{2} \varphi+E_{2} / E_{1} \sin ^{2}},
$$

где $E_{i}, \mu_{j}$ - физико-механические характеристики материала однонаправленной ленты.

Отметим, что при проектировании оболочки без учета работы связующего в материале однонаправленной ленты данное соотношение принимает вид

$$
\frac{N_{\beta}}{N_{\alpha}}=2-R_{2} / R_{1}=\operatorname{tg}^{2} \varphi=f(r) .
$$

B случае проектирования многослойной конструкции днища с разными углами армирования и различными материалами для определения коэффициентов $B_{i j}$ необходимо использовать общие соотношения для многослойных оболочек [2].

Используя соотношение

$$
\frac{d \sin \alpha}{\sin \alpha}=[2-f(r)] \frac{d r}{r},
$$

для построения формы днищ оболочки воспользуемся алгоритмом численного определения следующих функций:

$$
\begin{aligned}
\sin \alpha & =\sin \alpha_{0} e^{-\int_{R}^{r}(2-f(r) / r d r}, \\
y & =\int_{R}^{r} t g \alpha d r, \\
\frac{1}{R_{1}} & =\frac{d \sin \alpha}{d r}, \frac{1}{R_{2}}=\frac{\sin \alpha}{r} .
\end{aligned}
$$

В предположении, что силовая оболочка баллона образована намоткой $i$ слоев нитей с углами армирования $\pm \varphi_{i}$ и кольцевой подмоткой на цилиндрической части $\varphi=\pi / 2$, структура алгоритма выбора рациональной формы днищ оболочки при этом сводится к следующего рода действиям.

1. Задаются параметры оболочки $R_{l}, r_{0}$ где $R_{l,}-$ радиус цилиндрической части лейнера; $r_{0}-$ радиус полюсного отверстия лейнера.

2. Устанавливается величина расчетного давления $p: p_{r}=K p_{0}-$ минимальное давление разрушения; $p_{i}=1,5 p_{0}$ - испытательное давление; $p_{m}=\frac{2 \delta_{m} \bar{\sigma}_{t}^{m}}{d}$ или $p_{m}=\frac{4 \delta_{m} \bar{\sigma}_{t}^{m}}{\sqrt{3} d}-$ ми- ни мальноедавление, при котором происходит текучесть материала лейнера; $p=p_{r}-p_{m} \quad$ расчетное давление, где $p_{0}$ - рабочее давление в баллоне; $\delta_{m}-$ толщина стенки лейнера; $\bar{\sigma}_{t}^{m}-$ предел текучести материала лейнера; $K$ - коэффициент безопасности.

3. Назначаются армирующие материалы с различными линейными плотностями $N_{i}$, удельными плотностями материала $\rho_{i}$, прочностью $\sigma_{\dot{g}}$ и определяются площади эффективного сечения $f_{i}$ и разрывные нагрузки армирующих нитей (жгутов) $T_{i}$.

4. По известным зависимостям [2] определяются физико-механические характеристики $E_{1}^{i}, v^{i}{ }_{12}, v^{i}{ }_{21}, E_{2}^{i}, G_{12}^{i}$ для однонаправленного композитного материала (выбранной полоски) $i$-го слоя, где $E^{i}{ }_{1}, E^{i}{ }_{2}-$ модули упругости однонаправленного композита вдоль и поперек армирования; $G_{12}^{i}$ - модуль сдвига в плоскости армирования; $v^{i}{ }_{12}, v^{i} 21 \quad$ - коэффициенты Пуассона.

5. Задается некоторая поверхность и ее сечения $r_{01}, r_{02}, r_{03}, r_{0 n}$, которые определяют число слоев, образующих оболочку.

6. Задаются первоначальные значения радиусов мнимых цилиндрических поверхностей $R_{1}$, $R_{2}, R_{3}, R_{n}$, вложенных в оболочку с максимальным диаметром $R$. При этом учитывается условие, что $r_{i}+1<R_{i}<r_{i}+2$.

7. Из условия геодезического расположения нитей (жгутов) армирующих материалов по поверхности днища определяются углы армирования $\varphi_{i}$ на экваторе оболочки.

8. Последовательно начиная с первого участка $r_{0}-r_{1}$ для каждой мнимой оболочки с радиусом цилиндра $R_{i}$, используя соотношение

$$
T_{i} n_{i} \cos \varphi_{i}=\pi \int_{r_{0}}^{r_{i}} p r d r+Q_{1}-\sum_{j=1}^{i-1} T_{j} n_{j} \cos \varphi_{j}
$$

вычисляется число армирующих нитей(жгутов) $n_{i}$ в рассматриваемом слое.

9. На интервале изменения радиуса $R>r>r_{0}$ начиная от $r=R$ пошагово свыбранным интервалом $\Delta r$ изменения $r$ определяется [2] изменение толщины $h_{i}$ каждой зоны $i$

$$
h_{i}(r)=\frac{n_{s i} f_{i}}{2 \pi r \cos \varphi_{i}}
$$


с учетом особенностей в зоне $r_{0 i}$ и значения коэффициентов жесткости $B_{11}(r), B_{22}(r), B_{12}(r)$ с учетом $\bar{E}_{S}=\lambda \bar{E}_{S}^{0}$ и $h(r)=\sum_{i=1}^{k} h_{i}(r)$.

10. На том же интервале изменения радиуса $R>r>r_{0}$ строится численный алгоритм расчета и интегрирования функций (7).

11. Для окончательно выбранной схемы армирования на интервале изменения радиуса $R>r>r_{0}$ пошагово с выбранным интервалом $\Delta r$ изменения $r$ рассчитываются деформации $\varepsilon_{a}, \varepsilon_{\beta}$ и их интенсивность $\varepsilon_{i}$ в материале композитной оболочки и лейнера и с использованием критерия Коффина-Менсона [1] оценивается интервал живучести лейнера.

При неудовлетворительных результатах процесс повторяется с уменьшением ограничения по деформируемости оболочки баллона.

Для окончательно выбранной схемы армирования, используя метод ступенчатого нагружения, по известным методикам [3] определяются напряжения и деформации, возникающие в материалах конструкции при ее нагружении до уровня испытательного давления.

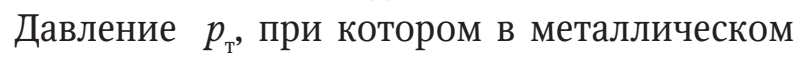
слое появляется пластическая деформация, оценивается из условия $\sigma_{i}=\sigma_{T}$, где интенсивность напряжений $\sigma_{T}$ определяется формулой:

$$
\sigma_{i}=\sqrt{\left(\sigma_{\alpha}^{m}\right)^{2}-\sigma_{\alpha}^{m} \sigma_{\beta}^{m}+\left(\sigma_{\beta}^{m}\right)^{2}+p\left(\sigma_{\alpha}^{m}+\sigma_{\beta}^{m}\right)+p^{2}} .
$$

При давлении $p>p_{\text {т }}$ для описания напряженно-деформированного состояния металлического слоя используются соотношения деформационной теории пластичности в форме метода переменных параметров упругости:

$$
\begin{gathered}
\sigma_{\alpha}^{m}=\bar{E}_{s}\left(\varepsilon_{\alpha}^{m}+v_{s} \varepsilon_{\beta}^{m}\right)-\frac{v_{s}}{1-v_{s}} p, \\
\sigma_{\beta}^{m}=\bar{E}_{s}\left(\varepsilon_{\beta}^{m}+v_{s} \varepsilon_{\alpha}^{m}\right)-\frac{v_{s}}{1-v_{s}} p
\end{gathered}
$$

где

$$
\bar{E}_{\mathrm{s}}=E_{5} /\left(1-v_{\mathrm{s}}^{2}\right), \quad v_{s}=\frac{1}{2}-\frac{E_{s}\left(\sigma_{i}\right)}{E}\left(\frac{1}{2}-v\right) .
$$

$E_{s}\left(\sigma_{i}\right) \quad-$ секущий модуль, зависящий от интенсивности напряжений; $v_{s}$ - пластический коэффициент Пуассона.
Для построения численного решения в данном случае водится степенная аппроксимация зависимости интенсивности напряжений от интенсивности деформаций для материала лейнера $\sigma_{i}=C \varepsilon_{i}^{n}$ где $C$ и $n$ - некоторые постоянные коэффициенты.

Отметим, что аппроксимация экспериментальной диаграммы деформирования осуществляется от деформации, при которой в металле появляется пластическая деформация, до деформации, при которой ожидается разрушение баллона (как правило, от 0,2 до 2,8 \%). Весь процесс расчета упругопластического решения строится на методе последовательных приближений.

Заключение. Рассмотрена постановка задачи рационального проектирования металлокомпозитного баллона давления, предполагающая определение формы образующей баллона, которая обеспечивает допустимый уровень деформируемости лейнера при заданном ресурсе эксплуатации баллона. Показано, что для окончательно выбранной схемы армирования используют метод ступенчатого нагружения с определением напряжения и деформации, возникающих в материалах конструкции при ее нагружении до уровня испытательного давления.

Настоящая статья выполнена в рамках договора № 02.G25.31.0175 «Разработка новых импортозамещающих технологий производства металлокомпозитных емкостей и баллонов высокого давления с применением высокоточного позиционирования заготовок, микроплазменной сварки и программно-аппаратной интегращии и оптимизации параметров технологического проиесса изготовления и испытаний», финансируемого Министерством образования и науки Российской Федерации.

\section{СПИСОК ЛИТЕРАТУРЫ}

1. Андреевская Г.Д. Высокопрочные ориентированные стеклопластики. - М.: Наука, 1966. - 371 с.

2. Ажогин Ф.Ф. Коррозионное растрескивание и защита высокопрочных сталей. - М.: Металлургия, 1974. -256 c.

3. Возможность и эффективность использования отечественных металлокомпозитных баллонов высокого давления в составе бортового оборудования воздушных судов / И.К. Лебедев [и др.] // Научный Вестник МГТУ ГА. - 2016. - № 2. - С. 137-142.

4. Конструкционные эпоксидные углепластики / Г.М. Гуняев [и др.] // Авиационная промышленность. - 1984. - № 12. - С. 41-45.

5. Лебедев И.К., Лебедев К.Н., Никонов В.В.. Технология и результаты испытаний нагруженного металлокомпозитного баллона высокого давления // Научный вестник ГосНИИ ГА. - 2017. - № 17. C. 90-96. 
6. Механические свойства сталей и сплавов при не стационарном нагружении: справочник / Д.А. Гохфельд [и др.]. - Екатеринбург: УрО РАН, 1996.

7. Тарнопольский Ю.М., Скудра А.М. Конструкционная прочность и деформативность стеклопластиков. - Рига, 1966. - 260 с.

8. Трощенко В.Т., Сосновский Л.А. Сопротивление усталости металлов и сплавов: справочник. Ч. 1. Киев: Наук, думка, 1987.

9. Фудзии Т., Дзако М. Механика разрушения композиционных материалов. - М.: Мир, 1982. - 232 с.

10. Черепанов Г.П. Механика разрушения композиционных материалов. - М.: Наука, 1983. - 296 с.

Лебедев Игорь Константинович, канд. техн. наук, ООО «Системы армированных фильтров и трубопроводов» («САФИТ»). Россия.
Мороз Николай Григорьевич, канд. техн. наук, ООО «Системы армированных фильтров и трубопроводов» («САФИТ»). Россия.

141351, Московская обл., Сергиево-Посадский $p$-н, д. Жучки, $2 \partial$.

Тел.: (495) 989-48-42.

Калинников Александр Николаевич, зав. лабораторией, Межотраслевой инжиниринговый центр «Композить России» МГТУ им. Н.Э. Баумана, ФГАОУ ВО «Московский государственный технический университет имени Н.Э. Баумана (национальный исследовательский университет)». Россия.

105005, г. Москва, ул. 2-я Бауманская, 5.

Тел.: (499) 263-63-91.

Ключевые слова: баллоны высокого давления; стеклопластиковая оболочка; высокопрочный углепластик; малоцикловая прочность; коррозионная усталость.

\section{ON AN ALGORITHM FOR DESIGNING MULTILAYER METAL COMPOSITE PRESSURE CYLINDERS WITH A THIN-WALLED LINER}

Lebedev Igor Konstantinovich, Candidate of Technical Sciences, JSC "Reinforced Filters and Pipelines Systems" (SAFIT). Russia.

Moroz Nikolai Grygoryevich, Candidate of Technical Sciences, JSC "Reinforced Filters and Pipelines Systems" (SAFIT). Russia.

Kalinnikov Alexander Nikolayevich, Head of laboratory, Intersectoral Engineering Center "Composites of Russia”, Bauman Moscow State Technical University. Russia.

Keywords: high-pressure bottles; glass-reinforced plastic shell; high-strength carbon-fiber-reinforced plastic; low-cycle strength; corrosive fatigue.
It is regarded the formulation of the problem of rational design of metal-composite pressure cylinder, involving the determination of the shape of the cylinder forming, which provides an acceptable level of deformability of the liner at a given service life of the cylinder. It is shown that for the final selected reinforcement scheme the method of step loading is used with the determination of the stress and strain arising in the materials of construction, when it is loaded to the level of the test pressure.

\section{ПРОБЛЕМЫ БЕЗОПАСНОСТИ НА ПОДЗЕМНОМ ТРУБОПРОВОДНОМ ТРАНСПОРТЕ И ТЕОРЕТИЧЕСКОЕ ОБОСНОВАНИЕ ИХ УСТРАНЕНИЙ}

Удк 658.382

университет

ОРЛОВ Павел Сергеевич, Ярославская государственная сельскохозяйственная академия

СОЦКАЯ Ирина Марковна, Ярославская государственная сельскохозяйственная академия

ПОПОВА Екатерина Сергеевна, Ярославская государственная сельскохозяйственная академия

ШКРАБАК Роман Владимирович, Санкт-Петербургский государственный аграрный

университет

Приведены результаты исследований по проблемам безопасности на подземном трубопроводном транспорте. Показано, ито основными причинами, снижающими безопасность использования подземного трубопроводного транспорта, являются износ магистральных трубопроводов и оборудования, а также коррозионные процессы. Разработаны технические решения для повышения надежности трубопроводно20 транспорта, его безопасности.

Введение. В России широко развита сеть подземного трубопроводного транспорта (водо- и теплопроводы, нефтепроводы, канализационные системы, газопроводы). Каждый из этих видов имеет свои особенности и характеризуется своими показателями опасности и вредности. В связи с этим генерируются профессиональные риски, связанные с тем, что на территории Российской Федерации функционирует более 3700 опасных производственных объектов нефтегазовых предприятий газоперекачивающих объ- ектов - компрессорных станций (КС) [6].

Почти половина всех магистральных газопроводов диаметром более 700 мм находится за Полярным кругом на территориях, на которых осуществляется только выпас оленей (Республика Коми - менее 1 чел. $/$ км$\left.^{2}\right)$. Южнее плотность сельского населения растет, и в Вологодской области составляет 1-5 чел./км²; в Костромской, Новгородской, Тверской и Ярославской областях 5-10 чел./км²; во Владимирской, Ивановской, Кировской, Ленинградской, Мос- 